\title{
THE CONE PERCOLATION MODEL ON GALTON-WATSON AND ON SPHERICALLY SYMMETRIC TREES
}

\author{
VALDIVINO V. JUNIOR, FÁBIO P. MACHADO, \\ AND KRISHNAMURTHI RAVISHANKAR
}

\begin{abstract}
We study a rumour model from a percolation theory and branching process point of view. The existence of a giant component is related to the event where the rumour, which started from the root of a tree, spreads out through an infinite number of its vertices. We present lower and upper bounds for the probability of that event, according to the distribution of the random variables that defines the radius of influence of each individual. We work with Galton-Watson branching trees (homogeneous and non-homogeneous) and spherically symmetric trees which includes homogeneous and $k$-periodic trees.
\end{abstract}

\section{INTRODUCTION AND BASIC DEFINITIONS}

Lebensztayn and Rodriguez [8], introduced a disk percolation model on general graphs where a reaction chain starting from the origin of the graph, based on independent copies of a geometric random variables, may lead to the existence of a giant component.

This line of research was continued by Junior et al [6] and [7], focusing on $\mathbb{N}$ and on the homogeneous tree respectively, studing a family of dependent long range (not necessarily homogeneous) percolation model. They studied the criticality of each model, presenting suficient conditions under which the processes reach a giant component with

Date: July 15, 2018.

2010 Mathematics Subject Classification. 60K35, 60G50.

Key words and phrases. epidemic model, galton-watson trees, rumour model, spherically symmetric trees.

Research supported by CNPq (310829/2014-3), FAPESP (09/52379-8), PNPDCapes 536114 and Simons Foundation Collaboration grant 281207. 
positive probability. Besides they presented bounds for the probability of having a giant component based on what they considered the radius of influence of each vertex of $\mathbb{N}$.

Gallo et al [4] computed precisely the probability of having a giant component for the homogeneous version of one of the models proposed in Junior et al [6], and obtained information about the distribution of the range of the cluster of the origin when it is finite. Besides that, they obtained a law of large numbers and a central limit theorem for the proportion of the cluster of the origin in a range of size $n$ as $n$ diverges. The key step of of proofs presented in Gallo et al [4] is to show that, in each model, the vertices belonging to the cluster of the origin can be related to a suitably chosen discrete renewal process. Related results have been obtained recently by Bertachi and Zucca [3]. All these research papers are to a different degree, stimulated by the seminal work of Benjamini and Schram [2] when they proposed the study of percolation theory beyond the nearest neighbor independent setup on $\mathbb{Z}^{d}$.

Here we focus on Galton-Watson, homogeneous, periodic and spherically symmetric trees in a process where the radius of influences is given by non-negative discrete random variables. In the paper we use the letter $R$ to refer to that random variable and to make formulas neater we define $p_{k}=\mathbb{P}(R=k)$ for $k=0,1, \ldots$ To avoid trivialities we assume throughout this paper that $p_{0} \in(0,1)$. A graph $G$ is said a tree if for any pair of its vertices there is one and only one path (a subset of edges) conecting them. By $|A|$ we denote the cardinality of $A$. The degree of a vertex is the cardinality of its set of neighbors. For 
two vertices $u, v$ let $d(u, v)$, be the distance between $u$ and $v$, that is the number of edges the path from $u$ to $v$ has.

Consider a tree $\mathbb{T}$ (a connected graph with no cycles) and its set of vertices $\mathcal{V}(\mathbb{T})$. Single out one vertex from $\mathcal{V}(\mathbb{T})$ and call this $\mathcal{O}$, the origin of $\mathcal{V}(\mathbb{T})$. For each two vertices $u, v \in \mathcal{V}(\mathbb{T})$, consider that $u \leq v$ if $u$ belongs to the path connecting $\mathcal{O}$ to $v$.

For a tree $\mathbb{T}$ and $n \geq 1$ we define

$$
\begin{gathered}
T^{u}:=\{v \in \mathcal{V}: u \leq v\} \\
T_{n}^{u}:=\left\{v \in T^{u}: d(v, \mathcal{O}) \leq d(u, \mathcal{O})+n\right\}
\end{gathered}
$$

and

$$
M_{n}(u):=\left|\partial T_{n}^{u}\right|:=\left|\left\{v \in T^{u}: d(v, \mathcal{O})=d(u, \mathcal{O})+n\right\}\right|
$$

As in Junior et al [7, we say that the process survives if the number of vertices involved is infinite. Otherwise we say the process dies out. Our main interest is to obtain resuts concerning whether the process has positive probability of involving an infinite set of individuals. Besides we present lower and upper bounds for the probability of that event, according to the distribution of the random variables that defines the radius of influence of each individual.

The paper is organized as follows. Sections 2, 3, 4, and 5 present the main results and specific setups and distributions for the Cone Percolation model on Homogeneous Trees, Periodic Trees, Spherically Symmetric Trees and Galton-Watson T rees respectively. Section 6 brings the proofs for the main results presented along sections 2, 3, 4] and 5 together with auxiliary lemmas and useful inequalities. 


\section{Homogeneous Trees}

Let us start off with a definition.

Definition 2.1. The Cone Percolation Model on $\mathbb{T}$.

Let $\left\{R_{v}\right\}_{\{v \in \mathcal{V}(\mathbb{T})\}}$ and $R$ be a set of independent and identically distributed random variables. Furthermore, for each $u \in \mathcal{V}(\mathbb{T})$, we define the random sets

$$
B_{u}=\left\{v \in \mathcal{V}(\mathbb{T}): u \leq v \text { and } d(u, v) \leq R_{u}\right\}
$$

With these sets we define the Cone Percolation Model on $\mathbb{T}$, the nondecreasing sequence of random sets $I_{0} \subset I_{1} \subset \cdots$ defined as $I_{0}=\{\mathcal{O}\}$ and inductively $I_{n+1}=\bigcup_{u \in I_{n}} B_{u}$ for all $n \geq 0$.

Definition 2.2. The Cone Percolation Model survival

Consider $I=\bigcup_{n \geq 0} I_{n}$ be the connected component of the origin of $\mathbb{T}$. Under the rumor process interpretation, $I$ is the set of vertices which heard the rumor. We say that the process survives if $|I|=\infty$, referring to the surviving event as $V$.

Definition 2.3. Rooted homogeneous trees

We say that a tree, $\mathbb{T}_{d}$, is homogeneous, if each one of its vertices has degree $d+1$. From $\mathbb{T}_{d}$ we define $\mathbb{T}_{d}^{+}$, a rooted homogeneous tree. Pick a $u \in \mathcal{V}\left(\mathbb{T}_{d}\right)$ such that $d(\mathcal{O}, u)=1$ and consider

$$
\begin{gathered}
\mathbb{T}_{d}^{+}(u)=\left\{v \in \mathcal{V}\left(\mathbb{T}_{d}\right): u \leq v\right\} \\
\mathbb{T}_{d}^{+}:=\mathbb{T}_{d} \backslash \mathbb{T}_{d}^{+}(u)
\end{gathered}
$$

Consider $\mathbb{P}_{+}$and $\mathbb{P}$ the probability measures associated to the processes on $\mathbb{T}_{d}^{+}$and $\mathbb{T}_{d}$ (we do not mention the random variable $R$ unless 
absolutely necessary). By a coupling argument one can see that for a fixed distribution of $R$

$$
\mathbb{P}_{+}(V) \leq \mathbb{P}(V)
$$

Furthermore, by the definition of $\mathbb{T}_{d}^{+}$and its relation with $\mathbb{T}_{d}$ we have that for a fixed distribution of $R$

$$
\mathbb{P}_{+}(V)=0 \text { if and only if } \mathbb{P}(V)=0 \text {. }
$$

Theorem 2.4. Consider a Cone Percolation Model on $\mathbb{T}_{d}$. Then, for $\mathbb{E}\left(d^{R}\right)<2-\frac{1}{d}$, we have

$$
\frac{d+\mathbb{E}\left(d^{R}\right)-p_{0}}{d\left[1-\mathbb{E}\left(d^{R}\right)+p_{0}\right]} \leq \mathbb{E}(|I|) \leq \frac{\mathbb{E}\left(d^{R}\right)+d-2}{2 d-1-d \mathbb{E}\left(d^{R}\right)}
$$

Example 2.5. Consider $R \sim \mathcal{B}(p)$, a radius of influence satisfying

$$
\mathbb{P}(R=1)=p=1-\mathbb{P}(R=0),
$$

with $p d<1$. Then we have

$$
\mathbb{E}(|I|)=\frac{1+p}{1-d p}
$$

Example 2.6. Consider $R \sim \mathcal{G}(1-p)$, a radius of influence satisfying

$$
\mathbb{P}(R=k)=(1-p) p^{k}, k=0,1,2, \ldots
$$

and assume also $p d<\frac{1}{2}$. So we have

$$
\frac{1-d p+p-p^{2}}{1-2 d p+d p^{2}} \leq \mathbb{E}(|I|) \leq \frac{1-d p-p}{1-2 d p} .
$$

That gives us a fairly sharp bound even when we pick $p$ and $d$ such that $p d$ is very close to $\frac{1}{2}$ as, for example, $p=10^{-6}$ and $d=499,000$. For these parameters we get $250.438 \leq \mathbb{E}(|I|) \leq 250.501$.

Example 2.7. For $R \sim \mathcal{B}(n, p)$, a radius of influence satisfying

$$
\mathbb{P}(R=k)=\left(\begin{array}{l}
n \\
k
\end{array}\right) p^{k}(1-p)^{n-k}, k=0,1, \ldots, n
$$


and $p<\frac{1}{d-1}\left[\sqrt[n]{\frac{2 d-1}{d}}-1\right]$, we have

$$
\frac{d+(d p+1-p)^{n}-(1-p)^{n}}{d\left[1-(d p+1-p)^{n}+(1-p)^{n}\right]} \leq \mathbb{E}(|I|) \leq \frac{(d p+1-p)^{n}+d-2}{2 d-1-d(d p+1-p)^{n}}
$$

Assuming

$$
d=1,000, n=2 \text { and } p=4 \times 10^{-4} \text { we have } 24.825 \leq \mathbb{E}(|I|) \leq 24.924 .
$$

Example 2.8. For $R \sim \mathcal{P}(\lambda)$, a radius of influence satisfying

$$
\mathbb{P}(R=k)=\frac{\exp (-\lambda) \lambda^{k}}{k !}, k=0,1,2, \ldots
$$

and $\lambda<\ln \left(\sqrt[d-1]{2-\frac{1}{d}}\right)$, we have

$$
\frac{d+e^{(d-1) \lambda}-e^{-\lambda}}{d\left[1-e^{(d-1) \lambda}+e^{-\lambda}\right]} \leq \mathbb{E}(|I|) \leq \frac{e^{(d-1) \lambda}+d-2}{2 d-1-d e^{(d-1) \lambda}} .
$$

In particular, if

$$
d=1,000 \text { and } \lambda=6 \times 10^{-4} \text {, we find } 5.613 \leq \mathbb{E}(|I|) \leq 5.625 .
$$

\section{Periodic Trees}

Definition 3.1. We define a $k$-periodic tree with degree $\tilde{d}=\left(d_{1}, \cdots, d_{k}\right)$, $d_{i} \geq 2$ for all $i=1,2, \cdots, k$, as tree such that for any vertex whose distance to the origin is $n k+i-1$ for some $n \in \mathbb{N}$ has degree $d_{i}+1$. We refer to this tree as $\mathbb{T}_{\tilde{d}}$.

A few useful quantities to present the results in this section are

$$
\begin{gathered}
d_{(i)}=\text { the } i \text {-th smallest value in } \tilde{d} \\
G=G(\tilde{d}):=\sqrt[k]{\prod_{j=1}^{k} d_{j}} \\
c_{0}:=1 \text { and } c_{i}:=\frac{\prod_{j=1}^{i} d_{(j)}}{\sqrt[k]{\prod_{j=1}^{k}\left(d_{j}\right)^{i}}}=\frac{\prod_{j=1}^{i} d_{(j)}}{G^{i}}, i=1, \cdots, k-1 ; \\
\bar{c}_{0}:=1 \text { amd } \bar{c}_{i}:=\frac{\prod_{j=k+1-i}^{k} d_{(j)}}{\sqrt[k]{\prod_{j=1}^{k}\left(d_{j}\right)^{i}}}=\frac{\prod_{j=k+1-i}^{k} d_{(j)}}{G^{i}}, i=1, \cdots, k-1 .
\end{gathered}
$$


Definition 3.2. For $i=1, \ldots, k$ and $R$, the radius of influence, we define

$$
I_{i}(R)= \begin{cases}1 & \text { if } R=n k+i \text { for some } n \in \mathbb{N} \\ 0 & \text { otherwise. }\end{cases}
$$

Besides, we define

$$
\underline{x}_{n, i}:=\left(\prod_{j=1}^{k} d_{j}\right)^{n} \prod_{j=1}^{i} d_{(j)} \text { for } i \neq 0, \underline{x}_{n, 0}:=\left(\prod_{j=1}^{k} d_{j}\right)^{n} \text { and } \underline{x}_{-1, i}:=0
$$

and

$$
\bar{x}_{n, i}:=\left(\prod_{j=1}^{k} d_{j}\right)^{n} \prod_{j=1}^{i} d_{(k+1-j)} \text { for } i \neq 0 \text { and } \bar{x}_{n, 0}:=\left(\prod_{j=1}^{k} d_{j}\right)^{n} .
$$

and

$$
h_{i}(R)=\left[\sum_{m=0}^{\left\lfloor\frac{R-i}{k}\right\rfloor-1} \sum_{j=0}^{k-1}\left(\underline{x}_{m, j}\right)^{-1}+\sum_{j=0}^{i-1}\left(\underline{x}_{\left\lfloor\frac{R-i}{k}\right\rfloor, j}\right)^{-1}\right] G^{R}
$$

Analogously to definition 2.3, we consider the Cone Percolation Model on $\mathbb{T}_{\tilde{d}}^{+}$. Relations analogous to $(\underline{2.2})$ and $(\underline{2.3})$ also holds between $\mathbb{T}_{\tilde{d}}$ and $\mathbb{T}_{\tilde{d}}^{+}$.

Theorem 3.3. Consider the Cone Percolation Model on $\mathbb{T}_{\tilde{d}}^{+}$with radius of influence $R$

(I) If

$$
\sum_{i=0}^{k-1} c_{i} \mathbb{E}\left(G^{R} I_{i}(R)\right)>1+p_{0}
$$

then, $\mathbb{P}_{+}(V)>0$,

(II) If

$$
\sum_{i=0}^{k-1} \bar{c}_{i} \mathbb{E}\left(h_{i}(R) I_{i}(R)\right) \leq 1
$$

then, $\mathbb{P}_{+}(V)=0$.

Corollary 3.4. Consider the Cone Percolation Model on $\mathbb{T}_{d}^{+}$(the d-dimensional rooted homogeneous tree) with radius of influence $R$ 
(I) If $\left(1-p_{0}\right) d>1$ then, $\mathbb{P}_{+}(V)>0$,

(II) If $\left(1-p_{0}\right) d \leq 1$ and $\mathbb{E}\left(d^{R}\right)>1+p_{0}$ then, $\mathbb{P}_{+}(V)>0$,

(III) If $\mathbb{E}\left(d^{R}\right) \leq 2-\frac{1}{d}$ then, $\mathbb{P}_{+}(V)=0$.

Let $\rho$ and $\psi$ be, respectively, the smallest non-negative root of the equations

$$
\begin{aligned}
& \sum_{i=0}^{k-1} \mathbb{E}\left(\rho^{c_{i} G^{R}} I_{i}(R)\right)+(1-\rho) p_{0}=\rho, \\
& \sum_{i=0}^{k-1} \mathbb{E}\left(\psi^{\lfloor\bar{c} h(R)\rfloor} I_{i}(R)\right)=\psi,
\end{aligned}
$$

Theorem 3.5. Consider the Cone Percolation Model on $\mathbb{T}_{\tilde{d}}^{+}$. Then,

$$
1-\rho \leq \mathbb{P}_{+}(V) \leq 1-\psi
$$

Theorem 3.6. For the Cone Percolation Model on $\mathbb{T}_{\tilde{d}}$ with radius of influence $R$, it holds that

$$
1-\sum_{i=0}^{k-1} \mathbb{E}\left(\rho^{M_{R}(\mathcal{O})} I_{i}(R)\right) \leq \mathbb{P}(V) \leq 1-\sum_{i=0}^{k-1} \mathbb{E}\left(\psi^{\left|T_{R}^{\mathcal{O}}\right|} I_{i}(R)\right) .
$$

Corollary 3.7. For the Cone Percolation Model on $\mathbb{T}_{d}$ (the d-dimensional homogeneous tree) with radius of influence $R$, it holds that

$$
1-\left(1-\rho^{\frac{d+1}{d}}\right) p_{0}-\mathbb{E}\left(\rho^{\frac{(d+1)}{d} d^{R}}\right) \leq \mathbb{P}(V) \leq 1-\mathbb{E}\left(\psi^{\frac{(d+1)}{d-1}\left(d^{R}-1\right)}\right)
$$

where $\rho$ and $\psi$ are the smallest non-negative root of the equations (3.1) and (3.2).

Example 3.8. Consider a Cone Percolation Model in $\mathbb{T}_{\tilde{d}}, \tilde{d}=(4,9)$ assuming $R \sim \mathcal{G}(1-p)$. From Theorem 3.3 and equation (2.3)

$$
0.078542 \leq \inf \{p: \mathbb{P}(V)>0\} \leq 0.097374
$$


Example 3.9. Consider a Cone Percolation Model in $\mathbb{T}_{\tilde{d}}$, with $\tilde{d}=$ $(12,15,16)$. Assuming $R \sim \mathcal{B}(3,0.1)$, from Theorem 3.6 we have,

$$
0.266557 \leq \mathbb{P}(V) \leq 0.266894 \text {. }
$$

\section{Spherically Symmetric Trees}

Definition 4.1. We say that a tree, $\mathbb{T}_{S}$, is spherically symmetric, if any pair of vertices at the same distance from the origin, have the same degree.

Note that periodic trees are a subclass of spherically symmetric tree and therefore the results will also apply to periodic trees. In the previous section we obtained stronger results using the particular properties of periodic trees.

From definition 2.1 we consider the Cone Percolation Model on $\mathbb{T}_{S}$.

Definition 4.2. Let us define for a tree $\mathbb{T}$

$$
\operatorname{dim} \inf \partial \mathbb{T}:=\lim _{n \rightarrow \infty} \min _{v \in \mathcal{V}} \frac{1}{n} \ln M_{n}(v) .
$$

Observe that

$$
\operatorname{dim} \inf \partial \mathbb{T}_{d}=\ln d
$$

Theorem 4.3. For a Cone Percolation Model in $\mathbb{T}_{S}$ and $R$, the radius of influence, $\mathbb{P}(V)>0$ if

$$
\lim _{n \rightarrow \infty} \sqrt[n]{\rho_{n}}>e^{-\operatorname{dim} \inf \partial \mathbb{T}_{S}}
$$

where

$$
\rho_{n}:=\prod_{k=0}^{n-1}\left[1-\prod_{i=0}^{k} \mathbb{P}(R<i+1)\right] .
$$

Lemma 6.8 shows that $\rho_{n}$ is as a lower bound of the probability that the process starting from any vertex $v$ reaches the vertices at $\partial T_{n}^{v}$, 
Corollary 4.4. For a Cone Percolation Model in $\mathbb{T}_{S}$ and $R$, a radius of influence satisfying $\mathbb{P}(R \leq k)=1$ for some $k \in \mathbb{N}, \mathbb{P}(V)>0$ if

$$
\operatorname{dim} \inf \partial \mathbb{T}_{S}>\ln \left[\frac{1}{1-\prod_{j=1}^{k} \mathbb{P}(R<j)}\right]
$$

Corollary 4.5. For a Cone Percolation Model in $\mathbb{T}_{S}$ and $R$, a radius of influence satisfying

$$
\mathbb{P}(R=k)=\frac{Z_{\alpha}}{(k+1)^{\alpha}}, k=1,2, \ldots
$$

if dim inf $\partial \mathbb{T}_{S}>0$, then $\mathbb{P}(V)>0$.

Example 4.6. Consider a Cone Percolation Model in $\mathbb{T}_{S}$ with $R \sim$ $\mathcal{B}(p)$.

- If $\operatorname{dim} \inf \partial \mathbb{T}_{S}>-\ln p$ then, $\mathbb{P}(V)>0$,

- If $\mathbb{T}_{S}=\mathbb{T}_{\tilde{d}}$ and $G(\tilde{d})>\frac{1}{p}$ then, $\mathbb{P}(V)>0$.

\section{Galton-Watson Branching Trees}

\subsection{Non Homogeneous Galton-Watson Branching Trees. Con-} sider a supercritical Galton-Watson branching process starting from a single progenitor such that each individual whose distance from the progenitor is $n$ has a random number of offspring (independet of everything else) with generating function $f_{n}(s)=\sum_{k=0}^{\infty} q_{n}(k) s^{k}$.

Let us define $F=\left\{\left(f_{n}, d_{n}\right)\right\}_{n \in \mathbb{N}}$ where $d_{n}=f_{n}^{\prime}(1) \in(0, \infty)$. This Galton-Watson branching process yields a random family tree $\mathbb{T}_{F}$. We are particularly interested in a supercritical Galton-Watson tree, on the event of non extinction (infinite trees). A sufficient condition for that is $\liminf _{n \rightarrow \infty} d_{n}>1$. 
Definition 5.1. For a supercritical Galton-Watson tree on $\mathbb{T}_{F}$, let us define

$$
D\left(\mathbb{T}_{F}\right):=\lim _{n \rightarrow \infty} \min _{i \in \mathbf{N}} \frac{1}{n} \ln \left[\prod_{l=i}^{i+n-1} d_{l}\right] .
$$

In particular, if $F$ is a sequence of generating functions of degenerated random variables $\left\{X_{n}\right\}_{n \geq 0}$ such that $X_{n}=a_{n}$ we have that $\mathbb{T}_{F}$ equals to a spherically symmetric tree $\mathbb{T}_{S}$ with probability 1 . Then, with probability 1

$$
D\left(\mathbb{T}_{F}\right)=\operatorname{dim} \inf \partial \mathbb{T}_{S}
$$

Theorem 5.2. For a Cone Percolation Model on $\mathbb{T}_{F}$ with a radius of influence $R, \mathbb{P}(V)>0$ if

$$
\lim _{n \rightarrow \infty} \sqrt[n]{\rho_{n}}>e^{-D\left(\mathbb{T}_{F}\right)}
$$

where

$$
\rho_{n}:=\prod_{k=0}^{n-1}\left[1-\prod_{i=0}^{k} \mathbb{P}(R<i+1)\right] .
$$

\subsection{Homogeneous Galton-Watson Branching Trees. Consider a} supercritical Galton-Watson branching process starting from a single progenitor such that each individual has a random number of offspring (independet of everything else) whose average is $d>1$. This process yields a random infinite family tree, known as a supercritical GaltonWatson tree $\mathbb{T}_{F}$, where $d_{n}=d$ for all $n \in \mathbb{N}$, on the event of non extinction.

Theorem 5.3. Consider the Cone Percolation Model on a homogeneous supercritical Galton-Watson branching tree with radius of influence $R$.

(I) If $\left(1-p_{0}\right) d>1$ then, $\mathbb{P}[V]>0$, 
(II) If $\left(1-p_{0}\right) d \leq 1$ and $\mathbb{E}\left(d^{R}\right)>1+p_{0}$ then, $\mathbb{P}[V]>0$,

(III) If $\mathbb{E}\left(d^{R}\right) \leq 2-\frac{1}{d}$ then, $\mathbb{P}[V]=0$.

(IV) For $\mathbb{E}\left(d^{R}\right)<2-\frac{1}{d}$ we have

$$
\frac{1}{1-\mathbb{E}\left(d^{R}\right)+p_{0}} \leq \mathbb{E}(|I|) \leq \frac{d-1}{2 d-1-d \mathbb{E}\left(d^{R}\right)} .
$$

\section{Proofs}

\subsection{Homogeneous Trees.}

Proof of Theorem 2.4

Let us define now two auxiliary branching process. For the first, $\left\{\mathcal{X}_{n}\right\}_{n \in \mathbb{N}}$, each individual has a number of offspring distributed as the random variable $X$, assuming values in $\left\{0, d, d^{2}, \ldots\right\}$ such that

$\mathbb{P}[X=0]=p_{o}, \mathbb{P}[X=d]=p_{1}, \cdots, \mathbb{P}\left[X=d^{k}\right]=p_{k}$ for all $k=1,2, \ldots$

In the second auxiliary process, $\left\{\mathcal{Y}_{n}\right\}_{n \in \mathbb{N}}$, each individual has a number of offsprings distributed as the random variable $Y$, assuming values in $\left\{0, d, d+d^{2}, \ldots, \sum_{i=1}^{k} d^{i}, \ldots\right\}$ such that

$\mathbb{P}[X=0]=p_{o}, \mathbb{P}[X=d]=p_{1}, \cdots, \mathbb{P}\left[X=\sum_{i=1}^{k} d^{i}\right]=p_{k}$ for all $k=1,2, \ldots$

These two processes provide convenient lower bounds $\left(\left\{\mathcal{X}_{n}\right\}_{n \in \mathbb{N}}\right)$ and upper bounds $\left(\left\{\mathcal{Y}_{n}\right\}_{n \in \mathbb{N}}\right)$ for our process. Suppose that $R_{v}=r$ for a fixed site $v$. Then the set of vertices activated by $v$ is $T_{r}^{v}$, whose cardinality is $\sum_{i=1}^{r} d^{i}$ vertices. The activation process will go on. The process $\left\{\mathcal{X}_{n}\right\}_{n \in \mathbb{N}}$ will only count on those $d^{k}$ which are at distance $r$ from $v$ (the set $\left.\partial T_{r}^{v}\right)$. By the other side, the process $\left\{\mathcal{Y}_{n}\right\}_{n \in \mathbb{N}}$ counts activation that will be made by all of them $\left(T_{r}^{v}\right)$, in addition to disregarding the fact that some vertice will experience multiple activations from sites belonging to $T_{r}^{v}$. 
For these processes the average number of offsprings are respectively $\mu_{X}=\mathbb{E}\left(d^{R}\right)-p_{0}$ and $\mu_{Y}=\frac{d}{d-1}\left[\mathbb{E}\left(d^{R}\right)-1\right]$. As $\mu_{X}<1$ and $\mu_{Y}<1$ by hypothesis, the expected values for the total number of individuals are respectively

$$
\frac{1}{1-\mu_{X}}=\frac{1}{1+p_{0}-\mathbb{E}\left(d^{R}\right)}
$$

and

$$
\frac{1}{1-\mu_{Y}}=\frac{d-1}{2 d-1-d \mathbb{E}\left(d^{R}\right)} .
$$

Using the fact that the root has degree $d+1$ we can modify the processes $\left\{\mathcal{X}_{n}\right\}_{n \in \mathbb{N}}$ and $\left\{\mathcal{Y}_{n}\right\}_{n \in \mathbb{N}}$ such that the offspring distributions for the first generation are respectively

$$
\begin{aligned}
& \mathbb{P}[X=0]=p_{0}, \\
& \mathbb{P}\left[X=(d+1) d^{k-1}\right]=p_{k} \text { for } k=1,2, \ldots
\end{aligned}
$$

and

$$
\mathbb{P}\left[Y=\frac{(d+1)\left(d^{k}-1\right)}{d-1}\right]=p_{k} \text { for } k=0,1,2, \ldots
$$

For these modified processes the total expected number of individuals are respectively

$$
\mathbb{E}\left(\left|I_{x}\right|\right)=\sum_{k=1}^{\infty}\left(\frac{(d+1) d^{k-1}}{1+p_{0}-\mathbb{E}\left(d^{R}\right)}+1\right) p_{k}+p_{0}=\frac{d+\mathbb{E}\left(d^{R}\right)-p_{0}}{d\left(1-\mathbb{E}\left(d^{R}\right)+p_{0}\right)}
$$

and

$$
\begin{aligned}
\mathbb{E}\left(\left|I_{y}\right|\right) & =\sum_{k=0}^{\infty}\left(\left[\frac{(d+1)\left(d^{k}-1\right)}{(d-1)}\right]\left[\frac{(d-1)}{2 d-1-d \mathbb{E}\left(d^{R}\right)}\right]+1\right) p_{k} \\
& =\frac{\mathbb{E}\left(d^{R}\right)+d-2}{2 d-1-d \mathbb{E}\left(d^{R}\right)} .
\end{aligned}
$$


Since the reasonings that justified the lower and upper bounds at the beginning of the proof are valid with this modification, we have that $\mathbb{E}\left(\left|I_{x}\right|\right) \leq \mathbb{E}(|I|) \leq \mathbb{E}\left(\left|I_{y}\right|\right)$ and the result follows.

\subsection{Periodic Trees.}

Consider a $k$-periodic tree whose degrees are $d_{1}+1, d_{2}+1, \cdots, d_{k}+1$ and for $i=1, \ldots, k-1$

$$
J_{i}=\left\{\left(j_{1}, \ldots, j_{k}\right), 1 \leq j_{1}<j_{2}<\cdots<j_{i} \leq k\right\}
$$

Let us define for $n \in \mathbb{N}$

$$
\begin{aligned}
A_{n k} & =\left\{\left(\prod_{j=1}^{k} d_{j}\right)^{n}\right\} \\
A_{n k+i} & =\left\{\left(\prod_{j=1}^{k} d_{j}\right)^{n} \prod_{l=1}^{i} d_{j_{l}},\left(j_{1}, \ldots, j_{i}\right) \in J_{i}\right\} \text { for } i=1, \ldots, k-1 .
\end{aligned}
$$

We claim that for all $n \in \mathbb{N}, k \in \mathbb{N}$ and $v \neq \mathcal{O}$ that

$$
\begin{aligned}
\min A_{n k+i} & =\underline{x}_{n, i}, \\
\max A_{n k+i} & =\bar{x}_{n, i}, \\
M_{n k+i}(v) & \in A_{n k+i} .
\end{aligned}
$$

Let

$$
y_{n, i}:=\sum_{m=0}^{n-1} \sum_{j=0}^{k-1}\left(\underline{x}_{m, j}\right)^{-1}+\sum_{j=0}^{i-1}\left(\underline{x}_{m, j}\right)^{-1}
$$

Lemma 6.1. Consider a $k$-periodic tree whose degrees are $d_{1}+1, d_{2}+$ $1, \cdots, d_{k}+1, d_{i} \geq 2$ for all $i=1,2, \cdots k$. Consider a vertex $v \neq \mathcal{O}$. Then

$$
\left|T_{n k+i}^{v}\right| \leq\left\lfloor y_{n, i} \cdot \bar{x}_{n, i}\right\rfloor .
$$




\section{Proof of Lemma 6.1}

Consider first the following set up: $R=k, d(\mathcal{O}, v)=m k$ for some $m \in \mathbb{N}$ and $\mathbb{T}_{\tilde{d}}$ such that $d_{i}=d_{(i)}$ for all $i=1, \cdots, k$. Then

$$
\left|T_{n k+i}^{v}\right|=\left|\bar{x}_{n, i}+\frac{\bar{x}_{n, i}}{d_{k}}+\frac{\bar{x}_{n, i}}{d_{k} d_{k-1}}+\cdots+\frac{\bar{x}_{n, i}}{\prod_{j=1}^{k} d_{j}}\right| .
$$

Consider now the case where $R=n k$ and $d(\mathcal{O}, v)=m k$ for $n, m \in \mathbb{N}$ and $\mathbb{T}_{\tilde{d}}$ such that $d_{i}=d_{(i)}$ for all $i=1, \cdots, k$. Then

$$
\begin{aligned}
\left|T_{n k+i}^{v}\right| & =\left|\bar{x}_{n, i}+\frac{\bar{x}_{n, i}}{d_{k}}+\frac{\bar{x}_{n, i}}{d_{k} d_{k-1}}+\cdot+\frac{\bar{x}_{n, i}}{\prod_{j=1}^{k} d_{j}}\right| \\
& +\left|\frac{\bar{x}_{n, i}}{\left(\prod_{j=1}^{k} d_{j}\right) d_{k}}+\cdots+\frac{\bar{x}_{n, i}}{\left(\prod_{j=1}^{k} d_{j}\right)^{2}}\right|+\cdots \\
& +\left|\frac{\bar{x}_{n, i}}{\left(\prod_{j=1}^{k} d_{j}\right)^{n-1} d_{k}}+\cdots+\frac{\bar{x}_{n, i}}{\left(\prod_{j=1}^{k} d_{j}\right)^{n}}\right| .
\end{aligned}
$$

Observe now that on any tree, for any $v_{r}$ such that $d\left(\mathcal{O}, v_{r}\right)=r$

$$
\left|T_{m}^{v_{r}}\right|=\sum_{j=1}^{m} M_{j}\left(v_{r}\right)=M_{m}\left(v_{r}\right)+\sum_{j=1}^{m-1} M_{m}\left(v_{r}\right) .\left[\prod_{i=1}^{j} M_{1}\left(v_{r+m-i}\right)\right]^{-1}
$$

Now consider only $R=n k+i$ for $n \in \mathbb{N}$ and $i=1, \cdots, k-1$. So, from (6.1), (6.2) and (6.3), it follows that

$$
\begin{aligned}
\left|T_{n k+i}^{v}\right| & \leq\left\lfloor\bar{x}_{n, i}+\frac{\bar{x}_{n, i}}{d_{(1)}}+\frac{\bar{x}_{n, i}}{d_{(1)} d_{(2)}}+\cdots+\frac{\bar{x}_{n, i}}{\prod_{j=1}^{k} d_{(j)}}+\cdots\right. \\
& \left.+\frac{\bar{x}_{n, i}}{\left(\prod_{j=1}^{k-1} d_{(j)}\right)^{n} d_{(1)}}+\cdots+\frac{\bar{x}_{n, i}}{\left(\prod_{j=1}^{k} d_{(j)}\right)^{n} \prod_{j=1}^{i} d_{(j)}}\right\rfloor \\
& =\left\lfloor y_{n, i} \cdot \bar{x}_{n, i}\right\rfloor .
\end{aligned}
$$

Let us define two auxiliary branching process, being the first one $\left\{\mathcal{X}_{n}\right\}_{n \in \mathbb{N}}$. This process is defined by a random variable $X$, assuming values in $\left\{\underline{x}_{n, i}, i=0, \ldots, k-1\right.$, and $\left.n=0,1, \ldots,(n, i) \neq(0,0)\right\} \cup\{0\}$ 
such that

$$
\begin{aligned}
\mathbb{P}[X=0] & =: p_{0}, \\
\mathbb{P}\left[X=\underline{x}_{n, i}\right] & =: p_{n k+i} \text { for } i=0, \ldots, k-1, \text { and } n=0,1, \ldots,(n, i) \neq(0,0)
\end{aligned}
$$

Its expected value is given by the following lemma

\section{Lemma 6.2.}

$$
\mathbb{E}[X]=\sum_{i=0}^{k-1} c_{i} \mathbb{E}\left[G^{R} I_{i}(R)\right]-p_{0}
$$

Proof of Lemma 6.2

$$
\begin{aligned}
\mathbb{E}(X) & =\sum_{n=1}^{\infty} \underline{x}_{n, 0} p_{n k}+\sum_{i=1}^{k-1} \sum_{n=0}^{\infty} \underline{x}_{n, i} p_{n k+i} \\
& =\sum_{i=0}^{k-1} c_{i} \sum_{n=0}^{\infty} \prod_{j=1}^{k}\left(\sqrt[k]{d_{j}}\right)^{n k+i} p_{n k+i}-p_{0} \\
& =\mathbb{E}\left[G^{R} I_{0}(R)\right]+\sum_{i=1}^{k-1} c_{i} \mathbb{E}\left[G^{R} I_{i}(R)\right]-p_{0} \\
& =\sum_{i=0}^{k-1} c_{i} \mathbb{E}\left[G^{R} I_{i}(R)\right]-p_{0} .
\end{aligned}
$$

and its probability generating function is given by

\section{Lemma 6.3.}

$$
\varphi_{X}(s)=\sum_{i=0}^{k-1} \mathbb{E}\left[s^{c_{i} G^{R}} I_{i}(R)\right]+(1-s) p_{0} .
$$


Proof of Lemma 6.3

$$
\begin{aligned}
\varphi_{X}(s) & =p_{0}+\sum_{n=1}^{\infty} s^{\underline{x}_{n, 0}} p_{n k}+\sum_{i=1}^{k-1} \sum_{n=0}^{\infty} s^{\underline{x}_{n, i}} p_{n k+i} \\
& =p_{0}+\sum_{n=1}^{\infty} s^{G^{n k}} p_{n k}+\sum_{i=1}^{k-1} \sum_{n=0}^{\infty} s^{c_{i} G^{n k+i}} p_{n k+i} \\
& =p_{0}-s p_{0}+\sum_{i=0}^{k-1} \sum_{n=0}^{\infty} s^{c_{i} G^{n k+i}} p_{n k+i} \\
& =(1-s) p_{0}+\sum_{i=0}^{k-1} \mathbb{E}\left[s^{c_{i} G^{R}} I_{i}(R)\right]
\end{aligned}
$$

The second auxiliary process is $\left\{\mathcal{Y}_{n}\right\}_{n \in \mathbb{N}}$, a branching process defined by a random variable $Y$, assuming values on $\left\{\left\lfloor y_{n, i} \bar{x}_{n, i}\right\rfloor, i=0, \ldots, k-\right.$ 1 , and $n=0,1, \ldots\}$ such that

$$
\mathbb{P}\left[Y=\left\lfloor y_{n, i} \bar{x}_{n, i}\right\rfloor\right]=p_{n k+i} \text { for } i=0,1, \ldots, k-1 \text { and } n=0,1, \ldots
$$

Its expected value satisfies

\section{Lemma 6.4.}

$$
\mathbb{E}[Y] \leq \sum_{i=0}^{k-1} \bar{c}_{i} \mathbb{E}\left[h_{i}(R) I_{i}(R)\right] .
$$

Proof of Lemma 6.4

$$
\begin{aligned}
\mathbb{E}(Y) & \leq \sum_{i=0}^{k-1} \sum_{n=0}^{\infty} y_{n, i} \bar{x}_{n, i} p_{n k+i} \\
& =\sum_{i=0}^{k-1} \sum_{n=0}^{\infty}\left[\sum_{m=0}^{n-1} \sum_{j=0}^{k-1}\left(\underline{x}_{m, j}\right)^{-1}+\sum_{j=0}^{i-1}\left(\underline{x}_{m, j}\right)^{-1}\right] \bar{x}_{n, i} p_{n k+i} \\
& =\sum_{i=0}^{k-1} \bar{c}_{i} \sum_{n=0}^{\infty}\left[\sum_{m=0}^{n-1} \sum_{j=0}^{k-1}\left(\underline{x}_{m, j}\right)^{-1}+\sum_{j=0}^{i-1}\left(\underline{x}_{m, j}\right)^{-1}\right] \prod_{j=1}^{k}\left(\sqrt[k]{d_{j}}\right)^{n k+i} p_{n k+i} \\
& =\sum_{i=0}^{k-1} \bar{c}_{i} \mathbb{E}\left[h_{i}(R) I_{i}(R)\right] .
\end{aligned}
$$

and its probability generating function is given by 


\section{Lemma 6.5.}

$$
\varphi_{Y}(s)=\sum_{i=0}^{k-1} \mathbb{E}\left[s\left\lfloor\bar{c}_{i} h_{i}(R)\right\rfloor I_{i}(R)\right] .
$$

Proof of Lemma 6.5

$$
\begin{aligned}
\varphi_{Y}(s) & =\sum_{i=0}^{k-1} \sum_{n=0}^{\infty} s^{\left\lfloor y_{n, i} \bar{x}_{n, i}\right\rfloor} p_{n k+i} \\
& =\sum_{i=0}^{k-1} \sum_{n=0}^{\infty} s^{\left\lfloor y_{n, i} G^{n k+i} \bar{c}_{i}\right\rfloor} p_{n k+i} \\
& =\sum_{i=0}^{k-1} \mathbb{E}\left[s^{\left\lfloor\bar{c}_{i} h_{i}(R)\right\rfloor} I_{i}(R)\right] .
\end{aligned}
$$

\section{Proof of Theorem 3.3}

By a coupling argument one can see that our process dominates (by (6.1) and $(\underline{6.3}))\left\{\mathcal{X}_{n}\right\}_{n \in \mathbb{N}}$. This process survives as long as $\mathbb{E}[X]>1$. Therefore from Lemma 6.2 our process survives if

$$
\sum_{i=0}^{k-1} c_{i} \mathbb{E}\left[G^{R} I_{i}(R)\right]>1+\mathbb{P}(R=0),
$$

proving (I).

By the other side, also by a coupling argument, our process is dominated (by (6.2) and (6.3) ) by $\left\{\mathcal{Y}_{n}\right\}_{n \in \mathbb{N}}$. That process dies out provided $\mathbb{E}[Y] \leq 1$ therefore from Lemma 6.4 our process dies out if

$$
\sum_{i=0}^{k-1} \bar{c}_{i} \mathbb{E}\left(h_{i}(R) I_{i}(R)\right) \leq 1,
$$

proving (II).

Proof of Theorem 3.5

In order to find the extinction probability of $\left\{\mathcal{X}_{n}\right\}_{n \in \mathbb{N}}$ (Grimmett and Stirzaker( [5, p.173]), let us consider the smallest non-negative root of 
the equation $\rho=\varphi_{X}(\rho)$. Therefore from Lemma 6.3

$$
\sum_{i=0}^{k-1} \mathbb{E}\left[\rho^{c_{i} G^{R}} I_{i}(R)\right]+(1-\rho) p_{0}=\rho
$$

and by construction of the processes, as $\mathbb{P}_{+}\left[V^{c}\right] \leq \rho$, we have that

$$
1-\rho \leq \mathbb{P}_{+}(V)
$$

In order to find the extinction probability of $\left\{\mathcal{Y}_{n}\right\}_{n \in \mathbb{N}}$ (Grimmett and Stirzaker [5, p.173]), let us consider the smallest non-negative root of the equation $\psi=\varphi_{Y}(\psi)$. Therefore from Lemma 6.5

$$
\left.\sum_{i=0}^{k-1} \mathbb{E}\left[\psi\left\lfloor\bar{c}_{i} h_{i}(R)\right\rfloor I_{i}(R)\right]\right)=\psi
$$

and by the construction of the processes, as $\mathbb{P}_{+}\left[V^{c}\right] \geq \psi$, we have that

$$
\mathbb{P}_{+}(V) \leq 1-\psi
$$

\section{Proof of Theorem 3.6}

Observe that except for the root, all vertices see towards infinity a

tree like $\mathbb{T}_{\tilde{d}}^{+}$. So, assuming $R_{\mathcal{O}}=n k+i$ the probability for the process to survive is greater or equal than the probability of the process to survive from at least one of the $M_{n k+i}(\mathcal{O})$ trees that have as root the furthest infected vertices. Now note that, still assuming $R_{\mathcal{O}}=n k+i$, the probability for the process to survive on $\mathbb{T}_{\tilde{d}}$ is smaller or equal than the probability for the process to survive from at least one of the $\left|T_{n k+i}^{\mathcal{O}}\right|$ vertices which are in the radius of influence $\left(R_{\mathcal{O}}\right)$ of the origin of the tree as if each one had its own tree. Then

$$
\mathbb{P}\left(V \mid R_{\mathcal{O}}=n k+i\right) \geq 1-\left(1-\mathbb{P}_{+}(V)\right)^{M_{n k+i}(\mathcal{O})} \geq 1-\rho^{M_{n k+i}(\mathcal{O})}
$$

and

$$
\mathbb{P}\left(V \mid R_{\mathcal{O}}=n k+i\right) \leq 1-\left(1-\mathbb{P}_{+}(V)\right)^{\left|T_{n k+i}^{\mathcal{O}}\right|} \leq 1-\psi^{\left|T_{n k+i}^{\mathcal{O}}\right|}
$$


Then,

$$
\begin{aligned}
\mathbb{P}(V) & =\sum_{i=0}^{k-1} \sum_{n=0}^{\infty} \mathbb{P}\left(V \mid R_{\mathcal{O}}=n k+i\right) p_{n k+i} \\
& \geq \sum_{i=0}^{k-1} \sum_{n=0}^{\infty}\left[1-\rho^{M_{n k+i}(\mathcal{O})}\right] p_{n k+i} \\
& =1-\sum_{i=0}^{k-1} \mathbb{E}\left[\rho^{M_{R}(\mathcal{O})} I_{i}(R)\right]
\end{aligned}
$$

and

$$
\begin{aligned}
\mathbb{P}(V) & =\sum_{i=0}^{k-1} \sum_{n=0}^{\infty} \mathbb{P}\left(V \mid R_{\mathcal{O}}=n k+i\right) \mathbb{P}\left(R_{\mathcal{O}}=n k+i\right) \\
& \leq \sum_{i=0}^{k-1} \sum_{n=0}^{\infty}\left[1-\psi^{\left|T_{n k+i}^{\mathcal{O}}\right|}\right] p_{n k+i} \\
& =1-\sum_{i=0}^{k-1} \mathbb{E}\left(\psi^{\left|T_{R}^{\mathcal{O}}\right|} I_{i}(R)\right) .
\end{aligned}
$$

\subsection{Spherically Symmetric Trees.}

Suppose we have a set of independent random variables $\left\{R_{v}\right\}_{\left\{v \in \mathcal{V}\left(\mathbb{T}_{S}\right)\right\}}$ distributed as $R$. Assume $\mathbb{P}(R=0)<1$.

For $u \leq v \in \mathcal{V}\left(\mathbb{T}_{S}\right)$, consider the event

$$
V_{u, v}: \text { Process starting from } u \text { reaches } v \text {. }
$$

For a fixed integer $n$, let $X_{0}^{n}=\{\mathcal{O}\}$. Besides, for $j=1,2, \ldots$ consider

$$
X_{j}^{n}=\bigcup_{u \in X_{j-1}^{n}}\left\{v \in \partial T_{n}^{u}: V_{u, v} \text { occurs }\right\} .
$$

Again, for all $j=1,2, \ldots$ consider

$$
Z_{j}^{n}=\left|X_{j}^{n}\right|
$$

So, for all fixed positive integer $n,\left\{Z_{j}^{n}\right\}_{j \geq 0}$ is a branching process dominated by the number of vertices $v \in \partial T_{j n}^{\mathcal{O}}$ which are activated. 
Lemma 6.6. Consider $n$ fixed. For $\mu_{j}$, the mean number of offspring of one individual of generation $j$ for the process $\left\{Z_{j}^{n}\right\}_{j \geq 0}$, it holds that

$$
\mu_{j}:=\mu_{j}^{n}=M_{n}(u) \rho_{j}^{(n)},
$$

where $\rho_{j}^{(n)}=\mathbb{P}\left(V_{u, v}\right)$, for any fixed pair $u \leq v$ such that $d(\mathcal{O}, u)=j n$ and $d(\mathcal{O}, v)=(j+1) n$.

Proof of Lemma 6.6

For fixed $j$ and $n$, consider for some $u$ such that $d(\mathcal{O}, u)=j n, \partial T_{n}^{u}=$ $\left\{v_{1}, v_{2}, \ldots, v_{M_{n}(u)}\right\}$. So we can write the number of offspring of $u$ as $\sum_{i=1}^{M_{n}(u)} I_{\left\{V_{u, v_{i}}\right\}}$. Taking expectation finishes the proof.

Lemma 6.7. Consider $n$ fixed and $\rho_{j}^{(n)}=\mathbb{P}\left(V_{u, v}\right)$, for any fixed pair $u \leq v$ such that $d(\mathcal{O}, u)=j n$ and $d(\mathcal{O}, v)=(j+1) n$,

$$
\rho_{j}^{(n)} \geq \prod_{k=0}^{n-1}\left[1-\prod_{i=0}^{k} \mathbb{P}(R<i+1)\right] .
$$

Proof of Lemma 6.8

For any fixed pair $u \leq v$ such that $d(\mathcal{O}, u)=j n$ and $d(\mathcal{O}, v)=(j+1) n$ we have that

$$
V_{u, v}=\bigcap_{k=0}^{n-1}\left[\bigcup_{i=0}^{k}\left\{R_{u(i)} \geq k+1-i\right\}\right]
$$

where $u(i)$ is the vertex from the path conecting $u$ to $v$ such that $d(\mathcal{O}, u(i))=j n+i$. From this follows

$$
\begin{aligned}
\rho_{j}^{(n)} & =\mathbb{P}\left(\bigcap_{k=0}^{n-1}\left[\bigcup_{i=0}^{k}\left\{R_{u(i)} \geq k+1-i\right\}\right]\right) \\
& \geq \prod_{k=0}^{n-1} \mathbb{P}\left(\bigcup_{i=0}^{k}\left\{R_{u(i)} \geq k+1-i\right\}\right) .
\end{aligned}
$$


The inequality is a consequence of the FKG inequality (N.Alon and J.Spencer [1, p.89]).

Proof of Theorem 4.3

Assume that $\operatorname{dim} \inf \partial \mathbb{T}_{S}>0$. Then, for all $\alpha \in\left(0, \operatorname{dim} \inf \partial \mathbb{T}_{S}\right)$ there exists $N=N(\alpha)$ such that for all $n \geq N$

$$
\min _{v \in \mathcal{V}} \frac{1}{n} \ln M_{n}(v)>\alpha
$$

where

$$
M_{n}(v) \geq e^{\alpha n} \text { for all } v \in \mathcal{V} \text { and } n \geq N
$$

From Souza \& Biggins $([9$, p.40]) a branching process in varying environments is uniformly supercritical if there exists constants $a>0$ and $c>1$ such that

$$
\prod_{k=i}^{j+i-1} \mu_{k} \geq a c^{j}, \text { for all } i \geq 0 \text { and } j \geq 0 .
$$

Observe that that condition holds if

$$
\liminf _{j \rightarrow \infty} \mu_{j}>1
$$

From Lemma 6.6 we have that for $n \geq N$

$$
\liminf _{j \rightarrow \infty} \mu_{j} \geq e^{\alpha n} \rho_{n}=\left(e \sqrt[n]{\rho_{n}}\right)^{n}
$$

Now note that we can write

$$
Z_{j+1}=\sum_{i=1}^{Z_{j}} Y_{j, i}^{n}
$$

where $Y_{j, i}^{n}$ are i.i.d. copies of $Y_{j}^{n}$, being the number of offspring from the $i-t h$ individual of the $j-t h$ generation. By considering Lemma 6.6 we have for all $j$ that

$$
\frac{Y_{j}^{n}}{\mu_{j}} \leq \frac{M_{n}(u)}{\mu_{j}}=\frac{1}{\rho_{j}^{(n)}} \leq(\mathbb{P}[R>0])^{-n}
$$


where $\rho_{j}^{(n)}=\mathbb{P}\left(V_{u, v}\right)$, for any fixed pair $u \leq v$ such that $d(\mathcal{O}, u)=j n$ and $d(\mathcal{O}, v)=(j+1) n$.

So, from Theorem 1 in Souza \& Biggins ([9, p.40]), we conclude that the cone percolation process has a giant component with positive probability if

$$
\lim _{n \rightarrow \infty} \sqrt[n]{\rho_{n}}>e^{-\alpha}
$$

As this hold for every $\alpha \in\left(0, \operatorname{dim} \inf \partial \mathbb{T}_{S}\right)$, the condition

$$
\lim _{n \rightarrow \infty} \sqrt[n]{\rho_{n}}>e^{-\operatorname{dim} \inf \partial \mathbb{T}_{S}}
$$

guarantees the survival of the process with positive probability.

Proof of Corollary 4.4

$$
\begin{aligned}
& \sqrt[n]{\prod_{i=0}^{n-1}\left[1-\prod_{j=0}^{i} \mathbb{P}(R<j+1)\right]=} \\
& =\left[1-\prod_{j=1}^{k} \mathbb{P}(R<j)\right] \sqrt[n]{\frac{\prod_{i=0}^{k-1}\left[1-\prod_{j=0}^{i} \mathbb{P}(R<j+1)\right]}{\left(1-\prod_{j=1}^{k} \mathbb{P}(R<j)\right)^{k}}} \\
& \rightarrow 1-\prod_{j=1}^{k} \mathbb{P}(R<j), \text { when } n \rightarrow \infty .
\end{aligned}
$$

Proof of Corollary 4.5

Observe that

$$
\begin{aligned}
\rho_{n} & \geq \mathbb{P}(R \geq n)=\sum_{k=n}^{\infty} \frac{Z_{\alpha}}{(k+1)^{\alpha}} \\
& \geq \int_{n+1}^{\infty} \frac{Z_{\alpha}}{x^{\alpha}} d x=\frac{Z_{\alpha}}{(\alpha-1)(n+1)^{\alpha-1}}
\end{aligned}
$$

The above inequalitty follows from the integral test.

Now observe that if $\operatorname{dim} \inf \partial \mathbb{T}_{S}>0$, we have that

$$
\lim _{n \rightarrow \infty} \sqrt[n]{\rho_{n}} \geq \lim _{n \rightarrow \infty} \sqrt[n]{\frac{Z_{\alpha}}{(\alpha-1)} \frac{1}{(n+1)^{\alpha-1}}}=1>e^{-\operatorname{dim} \inf \partial \mathbb{T}_{S}}
$$


Theorem 4.3 guarantess the desired result.

\subsection{Galton-Watson Branching Trees.}

\subsubsection{Non Homogeneous Galton-Watson Branching Trees.}

Proof of Theorem 5.2

Suppose we have a set of independent random variables $\left\{R_{n, m}\right\}_{\{n, m \in \mathbb{N}\}}$ distributed as $R$. Assume $\mathbb{P}(R=0)<1$. For each tree $\mathbb{T}_{f}$ on $\mathbb{T}_{F}$ we associate each of its existing vertices to a pair $u=(n, m)$ so that $R_{n, m}$ is its radius of influence. With this aim, $n$ stands for the distance from a set of $k(n)$ vertices to the tree progenitor while $m=1, \cdots, k(n)$ stands for an enumeration on the set of the existing vertices at level $n$.

For each tree $\mathbb{T}_{f}$ on $\mathbb{T}_{F}$ and $u \leq v \in \mathcal{V}\left(\mathbb{T}_{f}\right)$, consider the event

$$
V_{u, v}: \text { Process starting from } u \text { reaches } v \text {. }
$$

Let

$$
\Omega=\left\{\left(\mathbb{T}_{f} ;\left\{r_{n, m}\right\}_{\{n, m \in \mathbb{N}\}}\right) ; \mathbb{T}_{f} \in \mathbb{T}_{F} ;\left\{r_{n, m}\right\}_{\{n, m \in \mathbb{N}\}} \in \mathbb{N}^{\mathbb{N} \times \mathbb{N}}\right\}
$$

Take $\omega=\left(\mathbb{T}_{f} ;\left\{r_{n, m}\right\}_{\{n, m \in \mathbb{N}\}}\right)$. For a fixed integer $n$, let $X_{0}^{n}(\omega)=$ $\{\mathcal{O}\}$. Besides, for $j=1,2, \ldots$ consider

$$
X_{j}^{n}(\omega)=\bigcup_{u \in X_{j-1}^{n}(\omega)}\left\{v \in \partial T_{n}^{u}(\omega): I_{V_{u, v}}(\omega)=1\right\} .
$$

The definition for $\partial T_{n}^{u}(\omega)$ is analogous to (1.1). Again, for all $j=$ $1,2, \ldots$ consider

$$
Z_{j}^{n}=\left|X_{j}^{n}\right|
$$

So, for all fixed positive integer $n,\left\{Z_{j}^{n}\right\}_{j \geq 0}$ is a branching process dominated by the number of vertices $v \in \partial T_{j n}^{\mathcal{O}}$ which are activated. 
Lemma 6.8. Consider $n$ fixed. For $\mu_{j}$, the mean number of offspring of one individual of generation $j$ for the process $\left\{Z_{j}^{n}\right\}_{j \geq 0}$, it holds that

$$
\mu_{j}:=\mu_{j}^{n}=\left[\prod_{i=j n+1}^{j n+n} d_{i}\right] \rho_{j}^{(n)},
$$

where $\rho_{j}^{(n)}=\mathbb{P}\left(V_{u, v}\right)$, for any fixed pair $u \leq v$ such that $d(\mathcal{O}, u)=j n$ and $d(\mathcal{O}, v)=(j+1) n$.

\section{Proof of Lemma 6.8}

For fixed $j$ and $n$, consider for some $u$ such that $d(\mathcal{O}, u)=j n, \partial T_{n}^{u}=$ $\left\{v_{1}, v_{2}, \ldots, v_{M_{n}(u)}\right\}$. So we can write the number of offspring of $u$ as $\sum_{i=1}^{M_{n}(u)} I_{\left\{V_{u, v_{i}}\right\}}$, where $M_{n}(u)$ is a random variable. Note that $\mathbb{E}\left[M_{n}(u)\right]=$ $\prod_{i=j n+1}^{j n+n} d_{j}$. Taking expectation and using principle of substitution finishes the proof.

Proof of Theorem 4.3

Assume that $D\left(\mathbb{T}_{F}\right)>0$. Then, for all $\alpha \in\left(0, D\left(\mathbb{T}_{F}\right)\right)$ there exists $N=N(\alpha)$ such that for all $n \geq N$

$$
\min _{i \in \mathbf{N}} \frac{1}{n} \ln \left[\prod_{j=i+1}^{i+n} d_{j}\right]>\alpha
$$

where

$$
\prod_{j=i+1}^{i+n} d_{j} \geq e^{\alpha n} \text { for all } i \in \mathbf{N} \text { and } n \geq N .
$$

Now we write

$$
Z_{j+1}=\sum_{i=1}^{Z_{j}} Y_{j, i}^{n}
$$

where $Y_{j, i}^{n}$ are i.i.d. copies of $Y_{j}^{n}$, being the number of offspring from the $i-t h$ individual of the $j-t h$ generation. By considering Lemma 6.6 we have for all $j$ that

$$
\mathbb{E}\left[\frac{Y_{j}^{n}}{\mu_{j}}\right]=\frac{1}{\rho_{j}^{(n)}} \leq(\mathbb{P}[R>0])^{-n}
$$


where $\rho_{j}^{(n)}=\mathbb{P}\left(V_{u, v}\right)$, for any fixed pair $u \leq v$ such that $d(\mathcal{O}, u)=j n$ and $d(\mathcal{O}, v)=(j+1) n$.

Besides, by 6.5, Lemma 6.8 and Lemma 6.6

$$
\text { if } \lim _{n \rightarrow \infty} \sqrt[n]{\rho_{n}}>e^{-\alpha} \text { then } \liminf _{j \rightarrow \infty} \mu_{j}>1 .
$$

So, from Theorem 1 in Souza \& Biggins ([9, p.40]), we conclude that the cone percolation process has a giant component with positive probability if

$$
\lim _{n \rightarrow \infty} \sqrt[n]{\rho_{n}}>e^{-\alpha}
$$

As this holds for every $\alpha \in\left(0, D\left(\mathbb{T}_{F}\right)\right)$, the condition

$$
\lim _{n \rightarrow \infty} \sqrt[n]{\rho_{n}}>e^{-D\left(\mathbb{T}_{F}\right)}
$$

guarantees the survival of the process with positive probability.

\subsubsection{Homogeneous Galton-Watson Branching Trees.}

Proof of Theorem 5.3

Let us define two auxiliary branching process, being the first one $\left\{\mathcal{X}_{n}\right\}_{n \in \mathbb{N}}$. For this process,

$$
\mathbb{E}(\mathcal{X})=\sum_{n=0}^{\infty} \mathbb{P}(R=n) \mathbb{E}(\mathcal{X} \mid R=n)
$$

where

$$
\begin{aligned}
& \mathbb{E}(\mathcal{X} \mid R=0)=0 \\
& \mathbb{E}(\mathcal{X} \mid R=n)=d^{n}, \text { for } n=1,2, \ldots
\end{aligned}
$$

Note that

$$
\mathbb{E}(\mathcal{X})=\mathbb{E}\left[d^{R}\right]-p_{0}
$$

The second auxiliary process is $\left\{\mathcal{Y}_{n}\right\}_{n \in \mathbb{N}}$. For this process

$$
\mathbb{E}(\mathcal{Y})=\sum_{n=0}^{\infty} \mathbb{P}(R=n) \mathbb{E}(\mathcal{X} \mid R=n)
$$


where

$$
\mathbb{E}(\mathcal{Y} \mid R=n)=d+d^{2}+\cdots d^{n}
$$

Note that

$$
\mathbb{E}(\mathcal{Y})=\frac{d}{d-1}\left(\mathbb{E}\left[d^{R}\right]-1\right)
$$

Firstly we can assure (I) by a comparison with a supercritical branching process. In order to prove (II) and the left hand side one can see that our process dominates $\left\{\mathcal{X}_{n}\right\}_{n \in \mathbb{N}}$. This process survives as long as $\mathbb{E}[X]>1$ therefore from (6.6]) our process survives if $\mathbb{E}\left[d^{R}\right]>1+p_{0}$.

Secondly, also by a coupling argument, our process is dominated by $\left\{\mathcal{Y}_{n}\right\}_{n \in \mathbb{N}}$. That process dies out provided $\mathbb{E}[Y] \leq 1$ and $\mathbb{P}[Y=1] \neq 1$, therefore from (6.7) our process dies out if $\mathbb{E}\left[d^{R}\right] \leq 2-\frac{1}{d}$, proving (III) and the right hand side.

The proof of $(I V)$ follows from the fact that

$$
\frac{1}{1-\mathbb{E}[\mathcal{X}]} \leq \mathbb{E}[|I|] \leq \frac{1}{1-\mathbb{E}[\mathcal{Y}]}
$$

Acknowledgments: F.P.M. wishes to thank NYU-Shanghai China and V.V.J. and K.R. wish to thank Instituto de Matemática e EstatísticaUSP Brazil for kind hospitality.

\section{REFERENCES}

[1] N.Alon and J.Spencer The probabilistic method, (3rd ed.), Wiley, (2008).

[2] I.Benjamini and O.Schram. Percolation beyond $\mathbb{Z}^{d}$ : Many questions and a few answers, Elect. Comm. in Probab. 1 71-82, (1996).

[3] D.Bertacchi and F.Zucca. Rumor processes in random environment on $\mathrm{N}$ and on Galton-Watson trees. J. Stat. Phys., 153, (3), 486-511, (2013).

[4] S.Gallo, N.Garcia, V.Junior, P.Rodríguez Rumor processes on N and discrete renewal processes. J. Stat. Phys. 155, (3), 591-602, (2014).

[5] G.Grimmett and D.Stirzker. Probability and Random Processes, (3rd ed.), Oxford University Press, (2001).

[6] V.Junior, F.Machado and M.Zuluaga. Rumour Processes on $\mathbb{N}$, Journal of Applied Probability, 48 (3) 624-636, (2011). 
[7] V.Junior, F.Machado and M.Zuluaga. The Cone Percolation on $\mathbb{T}_{d}$, Brazilian Journal of Probability and Statistics, 28 (3) 367-675, (2014).

[8] E.Lebensztay and P.Rodriguez. The disk-percolation model on graphs, Statistics \& Probability Letters, 78, 14, 2130-2136, (2008).

[9] J.C.D'Souza and J.D.Biggins. The supercritical Galton-Watson process in varying environments, Stochastic Process. Appl. 42 (1), 39-47, (1992)

(Fábio P. Machado) Institute of Mathematics and Statistics, University of São Paulo, Rua do Matão 1010, CEP 05508-090, São Paulo, SP, BRAZIL - FMACHADO@IME.USP.BR

(Valdivino V. Junior) Federal University of Goias, Campus Samambaia, GoiÂNiA, GO, BRAZIL - VVJunior@UfG.BR

(Krishnamurthi Ravishankar) NYU-ECNU Institute of Mathematical SCIences at NYU Shanghai, 3663 Zhongshan Road North, Shanghai 200062 and 1555 Century Ave, Pudong, Shanghai, China - Kr26@Nyu.edu 